\title{
Tenodermodesis in the Treatment of Extensor Tendon Injury Resulting in Mallet Deformity of the Distal Interphalangeal Joint
}

\author{
Qasim Malik*, Shoaib Khan, Sophie Miller and Mohammad Waseem \\ Department of Trauma and Orthopaedics, Macclesfield District General Hospital, \\ $U K$ \\ *Corresponding Author: Qasim Malik, Registrar Trauma and Orthopaedics, \\ North Cumbria NHS Trust, UK.
}

Received: April 22, 2020

Published: June 01, 2020

(C) All rights are reserved by Qasim Malik, et al.

\section{Abstract}

Mallet injuries are treated surgically when conservative treatment fails to achieve the optimum outcome. Tenodermodesis without $\mathrm{K}$ wire fixation is a simple and safe surgical option for the management of acute and chronic Mallet injuries.

This study aims to assess the functional outcome of Mallet injuries treated with Tenodermodesis.

Eight patients were treated with Tenodermodesis in our centre over a period of 45 months for Mallet injury. Seven cases had closed injury, and one patient suffered an open Mallet injury. Closed injuries had Tenodermodesis following failed conservative treatment. The open injury was treated with wound debridement and Tenodermodesis as an emergency procedure. All patients were assessed with Quick DASH score for functional outcome along with patient satisfaction.

In our series, we lost two patients in follow up. Mean follow up was four months [range 2 - 8 months]. Five patients were satisfied with the outcome of the technique. One patient has superficial wound infection, and one had arthrodesis of distal interphalangeal joint because of persistent extension lag at distal interphalangeal joint. Average Quick DASH score was 7.20 (range 2.27 - 13.64).

We achieved a good functional outcome in our series with Tenodermodesis for the management of Mallet injury. There were no significant complications noted using this technique.

Keywords: Mallet Injury; Tenodermodesis; Splinting; Distal Interphalangeal Joint; DASH Score

\section{Abbreviations}

DASH Score: The Disabilities of the Arm, Shoulder and Hand Score DIPJ: Distal interphalangeal joint

\section{Introduction}

Mallet finger is the consequence of the extensor mechanism breach near its distal insertion, which results in extension loss at the distal interphalangeal joint [1]. Mallet fingers can have soft tissue component involving separation of the tendon at its insertion or bony element where the articular surface of the dorsal distal phalanx is fractured [1]. The goals of treatment in Mallet injuries is to achieve healing of the tendon in an anatomical position, maintain congruency of the DIP joint and minimise extension lag [1].

This injury is usually instigated by suddenly applied, forced flexion to an extended fingertip [1]. Rarely, Mallet injury can result from laceration and is managed by repair of the tendon and Kirschner wire fixation of distal interphalangeal joint [8].
Extensor tendon injuries of Mallet type has a prevalence of $9.3 \%$ of all tendon injuries. In tendon injuries of hand and wrist, its incidence is $5.6 \%$ [2].

There is no gender difference in the population affected by Mallet injury, albeit young males have high-energy, and elderly females have low-energy mechanisms of injury [3]. Usually, the dominant hand is affected $[4,5]$.

There is variability in the literature with regards to which finger is involved in this injury. Some authors have reported middle finger to be the most affected [4], others ring [6] and few little fingers [5]. Majority of Mallet injuries result from avulsion of the tendon with no bony involvement [7] and these injuries are treated by early splinting of the DIP joint [8].

In cases where the fracture of distal phalanx involves more than one-third of the articular surface, open reduction internal fixation is a better option of treatment [8]. Indications for surgery are disabling loss of extension, swan-neck deformity causing pain, or cosmesis [8]. 
Mallet finger with articular surface involvement of less than one third is managed with uninterrupted splinting $[9,10]$. Splinting is recommended to be applied continuously for the duration of six weeks, followed by night splinting for two to six weeks and during vigorous activities $[11,12]$.

Conservative treatment has given satisfactory outcomes and is generally preferred by most authors. Indications for surgical intervention include open injuries, patients unable to tolerate splinting and dorsally based distal phalanx fracture with palmar subluxation $[13,14]$.

Tenodermodesis has been used for the management of chronic mallet deformities where closed reduction and splinting failed, leaving patients with deformity and extension lag at the distal interphalangeal joint [8]. The technique promotes fibrous tissue formation between extensor and dermal layers, facilitating deformity correction and restoration of function [22].

\section{Purpose of the Study}

The purpose of our study evaluates tenodermodesis for an extensor tendon injury resulting in Mallet deformity which failed conservative treatment.

\section{Materials and Methods}

Eight patients were treated with Tenodermodesis in our centre over a period of 45 months. There were five females and three males. The average age was 54 [range 23.1 - 81.9]. Dominant hand was injured in 5 cases. The little finger was involved in 4 patients, Middle in 2 and Ring in 2. Seven cases had a blunt injury to the finger, and one had a laceration. Two patients had fracture associated with Mallet injury, and six were soft tissue injuries only. All patients with closed injuries were treated conservatively with a splint for an average of 8 weeks [range 6 - 10 weeks]. One patient who had open Mallet injury was treated as an emergency procedure with wound debridement and Tenodermodesis. Preoperatively all patients had full range of movement at distal interphalangeal joint passively. Radiographs of the involved finger revealed no evidence of distal interphalangeal joint subluxation. Quick DASH score was used to gauge improvement in patient pain, disability, and high-performance activities.

Quick DASH score consists of 11 items which are derived from Disabilities of arm, shoulder, and hand score [DASH]. It is a shorter version of the 30 item DASH score [15]. It is less cumbersome for the responder and easy to interpret for analysis. It has been reliable in several studies, and the results have shown that it is more precise with good validity and reliability compared to the DASH score [16]. A study conducted to compare data collection through verbal and self-administered written quick DASH score showed good test-retest performance and provided flexibility in data collection for the researcher [17].

\section{Surgical technique}

All the patients were consented after explaining the risks and benefits of the surgical procedure. The injured finger was marked as recommended by the World Health Organisation [WHO] checklist. A finger tourniquet was applied to all patients [figure 1-a]. The hand was prepped and draped, followed by an elliptical incision over the dorsal surface of the distal interphalangeal joint. Extensor tendon was identified, and torn edges were debrided. A nonabsorbable, monofilament suture 2.0 proline was passed through the proximal edge of the wound taking the full thickness of skin and the free edge of the ruptured extensor tendon. The suture is then passed through the distal end of the wound taking deep capsular layer, tendon edge and the skin. A second and third suture was placed at an equal and symmetrical position, depending on the length of the incision. An assistant was asked to hold the distal phalanx in a reduced position to allow maximum approximation of the tendon edges while sutures were tied. This technique achieved repair of the extensor tendon in a single layer and permitted healing by fibrosis [figure 1-b]. The wound was washed out with saline, a simple sterile dressing was applied, and the tourniquet removed at the end of the procedure. The repair was protected with a mallet splint postoperatively for six weeks. After six weeks patients were advised to use the splint for another four weeks only during the night. Patients were seen in the clinic in two weeks for a wound check and then reviewed at regular intervals to monitor their progress. Sutures were retained for an average duration of six weeks. After removal of sutures, patients were referred to a hand therapist. In our centre, hand therapists work in close association with the Hand Surgeons and in case of any issues they can liaise with the Consultant to obtain advice regarding patient management.
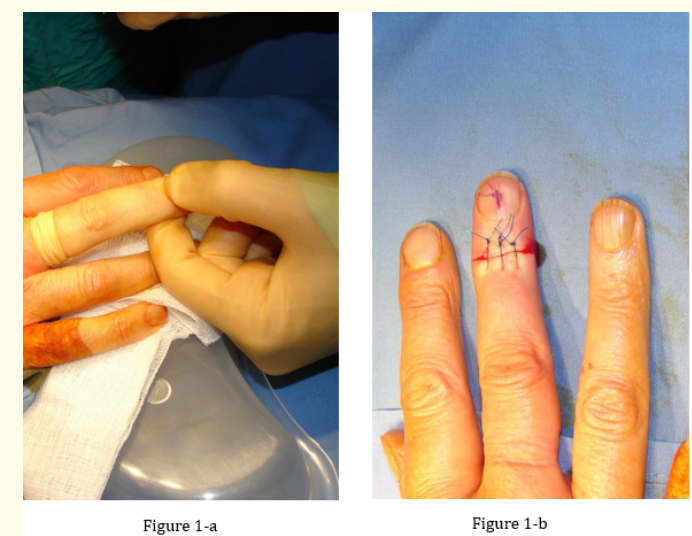

Figure 1a: Established mallet finger deformity. Ring tourniquet applied .

Figure 1b: Through and through 2.0 proline suture promoting healing of extensor tendon by fibrosis of all layer 


\section{Results and Discussion}

Mean follow up of our patients was four months [range 8 - 2 months]. Two cases were lost to follow up. 5 out of 6 patients were satisfied with the outcome of the surgery and had no restriction in daily activities of life. They were gratified with the appearance of the finger. Postoperative average Quick DASH score was 7.20 [range 2.27 - 13.64]. Mean postoperative Extension lag was $13.8^{\circ}$. Mean flexion at the distal interphalangeal joint was $87.5^{\circ}$. One patient suffered from superficial wound infection, which was treated with oral antibiotics. This patient had closed Mallet injury. One patient did not regain extension following the procedure, and because of disabling symptoms, an arthrodesis of the distal interphalangeal joint was performed. Due to poor outcome, this patient was not satisfied with the results of the intervention.

Mallet finger is due to the loss of extensor mechanism at the distal interphalangeal joint, also called a drop finger or baseball finger. Disruption in tendon substance and avulsion in bone have been reported as a cause of mallet finger. Uncomplicated cases are best managed by splinting therapies, whereas surgical interventions are best treatment if splinting therapy does not show any improvement.

Our case series revealed good satisfaction rate in patients treated with Tenodermodesis. All the patients had improvement in pain, disability, and high-performance function apart from one patient, who had no healing from this technique. This was managed with arthrodesis of the distal interphalangeal joint because of persistent disability.

We followed the surgical technique which involved approximation of the skin, extensor tendon edges and part of the dorsal capsule with non-absorbable suture. This technique enhanced healing of the tendon by providing an appropriate approximation of the tendon edges, which is sometimes difficult to achieve in conservative treatment. The stitches were kept for the longer duration, therefore, providing optimal time for the process of fibrosis to complete before flexion of the distal interphalangeal can be commenced. Further protection of the repair was accomplished by splinting of the distal interphalangeal joint postoperatively.

Islen., et al. utilised tenodermodesis for the repair of the injury and avoided fixation with $\mathrm{K}$ wire. Their technique involved resection of skin, tendinous scar tissue and dorsal capsule elements if it was impinging in the joint space. They approximated the skin, tendon and capsular edges using 3 to 4 absorbable monofilament sutures which were $2 \mathrm{~mm}$ apart. Tying the sutures was able to correct extension lag [18]. Our technique was similar, but we utilised non-absorbable sutures to accomplish extension at the distal in- terphalangeal joint. Their postoperative protocol was to keep the joint in full extension with a digital splint which was worn by the patients for 35 days and night splint was used until the sixtieth day [18]. Their prerequisites for the procedure were a good quality of tendon so it can hold the sutures and the full passive mobility of the joint. In their series of 26 cases, 22 had a satisfactory outcome with an average active range of motion of 10 - 60 degrees [18].

Nakamura and Nanjyo suggested open surgical repair for acute open mallet injuries. Their technique was based on using the figure of eight stainless steel wire to approximate the tendon stumps, which were then sutured with nylon. K-wire immobilisation of the DIP joint was done following the repair, which was continued for three weeks [19]. Our technique utilised non-absorbable suture for the repair of the tendon, and we did not immobilise the distal interphalangeal joint with $\mathrm{K}$ wire instead used splint postoperatively. In their series, they had three patients with open mallet injury inflicted by a knife and 12 patients with closed injuries. At one year follow up, mean extension deficit of 6 degrees, mean flexion angle of 64 degrees and active range of motion of 58 degrees was accomplished by their technique [19]

Doyle proposed a combination approach with surgical repair and splinting for open mallet injuries. He utilised a running suture for the approximation of both skin and tendon pursued by an extension splint. Sutures were removed after 10 to 12 days and the splint was continued for six weeks [20]. Our technique is much similar to Doyle apart from longer duration of sutures retention, and we used extension splint for six weeks as well.

Kon and Bloem utilised tenodermodesis for the repair of Mallet injury [21]. In their technique, they resected skin, tendinous scar tissue and part of the dorsal capsule. Three or four non-absorbable sutures were inserted through the skin, tendon, and capsule. Kirschner wire was used for fixation of distal interphalangeal joint followed by tying of sutures. Sutures were removed at $10^{\text {th }}$ postoperative day and Kirschner wire after six weeks. This technique is similar to the method used in our series, and we kept sutures for a longer duration. Instead of $\mathrm{K}$ wire, we used a splint for postoperative immobilisation. They reported a series of 27 patients and 26 had excellent results with an average range of motion close to normal [5 - 60 degrees]. They had two complications; one was a low-grade infection which required early removal of $\mathrm{K}$ wire and splint was utilised for the remaining duration for immobilisation. This complication did not interfere with the satisfactory outcome of the procedure. The second patient had an avulsion fracture of the distal phalanx and tenodermodesis failed to improve the deformity at distal interphalangeal joint [21]. 
Sorene., et al. performed tenodermodesis and $\mathrm{K}$ wire fixation of DIP for established mallet injuries which failed the trial of conservative treatment with splinting for 12 weeks. Their technique was similar to other authors in passing the sutures through skin, tendon and dorsal capsule for approximation. $\mathrm{K}$ wire was inserted through the distal interphalangeal joint and sutures were tied. Immobilisation of the distal interphalangeal joint was carried out for a month [8].

We had no difference in comparison to the technique of Sorene., et al. apart from the utilisation of splint rather than $\mathrm{K}$ wire in postoperative immobilisation. They had a series of 16 patients and reported excellent results in eight patients, good in six and fair in two. In their series, patients had a mean improvement in extension lag from 50 to 9 degrees [8].

Our technique avoided the use of $\mathrm{K}$ wire in achieving extension at the distal interphalangeal joint, which prevented the morbidity associated with insertion, retention and removal of $\mathrm{K}$ wire. It also reduces the risk of infection linked with metalwork placement.

\section{Conclusion}

Tenodermodesis is a simple procedure which can be completed in a short duration of time. There are no significant complications post-surgery. In several studies, it has shown good functional outcome. In our study, we found this technique useful in the treatment of chronic and acute, open mallet injuries. It allows early splint removal, acceptable cosmesis and function. We recommend its consideration in cases where conservative treatment has failed to achieve optimum outcome.

\section{Conflict of Interest}

No conflict of interest.

\section{Bibliography}

1. Bloom Jacob MP., et al. "Current concepts in the evaluation and treatment of mallet finger injury". Plastic and Reconstructive Surgery 132.4 (2013): 560e-566e.

2. De Jong Johanna P., et al. "The incidence of acute traumatic tendon injuries in the hand and wrist: a 10-year populationbased study". Clinics in Orthopedic Surgery 6.2 (2014): 196202.

3. Clayton Robert AE and Charles M Court-Brown. "The epidemiology of musculoskeletal tendinous and ligamentous injuries". Injury 39.12 (2008): 1338-1344.

4. Stark Herbert H., et al. "Mallet finger". Journal of Bone and Joint Surgery 44.6 (1962): 1061-1068.
5. Stern Peter J and John J Kastrup. “Complications and prognosis of treatment of mallet finger". The Journal of Hand Surgery 13.3 (1988): 329-334.

6. Robb WAT. "The results of treatment of mallet finger". The Journal of Bone and joint Surgery 41.3 (1959): 546-549.

7. Wehbé MA and LH Schneider. "Mallet fractures". The Journal of bone and joint surgery 66.5 (1984): 658-669.

8. Sorene Elliot and David Goodwin. "Tenodermodesis for established mallet finger deformity". Scandinavian Journal of Plastic and Reconstructive Surgery and Hand Surgery 38.1 (2004): 4345 .

9. Garberman Scott F., et al. "Mallet finger: results of early versus delayed closed treatment". Journal of Hand Surgery 19.5 (1994): 850-852.

10. Stack HG. "A modified splint for mallet finger". Journal of Hand Surgery 11.2 (1986).

11. Hovgaard C and B Klareskov. "Alternative conservative treatment of mallet-finger injuries by elastic double-finger bandage". Journal of Hand Surgery 13.2 (1988): 154-155.

12. Simpson D., et al. "Mallet deformity in sport". Journal of Hand Surgery 26.1 (2001): 32-33.

13. Bauze A and GI Bain. "Internal suture for mallet finger fracture”. Journal of Hand Surgery 24.6 (1999): 688-692.

14. Warren RANRM Kay and DG Ferguson. "Mallet finger: comparison between operative and conservative management in those cases failing to be cured by splintage". Journal of Hand Surgery 13.2 (1988): 159-160.

15. Beaton Dorcas E., et al. "Development of the QuickDASH: comparison of three item-reduction approaches". Journal of Bone and Joint Surgery 87.5 (2005): 1038-1046.

16. Gummesson Christina., et al. "The shortened disabilities of the arm, shoulder and hand questionnaire (Quick DASH): validity and reliability based on responses within the full-length DASH". BMC Musculoskeletal Disorders 7.1 (2006): 44.

17. London Daniel A., et al. "Performance characteristics of the verbal QuickDASH”. The Journal of hand Surgery 39.1 (2014): 100-107.

18. Iselin François., et al. "A simplified technique for treating mallet fingers: tenodermodesis". Journal of Hand Surgery 2.2 (1977): 118-121. 
19. Nakamura Kiyoshi and Bunshyo Nanjyo. "Reassessment of surgery for mallet finger". Plastic and Reconstructive Surgery 93.1 (1994): 141-149.

20. Doyle JR. "Extensor tendons-acute injuries methods of treatment of acute boutonniere deformity". Green's Operative Hand Surgery (1999): 1972-1974.

21. Kon M and JJAM Bloem. "Treatment of mallet fingers by tenodermodesis”. Hand 2 (1982): 174-176.

22. Smit Jeroen M., et al. "Treatment options for mallet finger: a review". Plastic and Reconstructive Surgery 126.5 (2010): 1624-1629.

\section{Assets from publication with us}

- Prompt Acknowledgement after receiving the article

- Thorough Double blinded peer review

- Rapid Publication

- Issue of Publication Certificate

- High visibility of your Published work

Website: https://www.actascientific.com/

Submit Article: https://www.actascientific.com/submission.php Email us: editor@actascientific.com

Contact us: +919182824667 